\title{
RESOLUCIÓN DE PROBLEMAS BASADA EN EL ANÁLISIS. HACER DEL ANÁLISIS Y DEL RAZONAMIENTO EL FOCO DE LA ENSEÑANZA DE LA FÍSICA
}

\author{
LEONARD WILLIAM, J. ${ }^{1}$, GERACE, WILLIAM, J. y DUFRESNE, ROBERT, J. \\ Scientific Reasoning Research Institute and Department of Physics \\ University of Massachusetts, Amherst, MA 01003-4525, EEUU \\ ${ }^{1}$ http://umperg.physics.umass.edu/
}

\begin{abstract}
Resumen. Usando los resultados provenientes de la investigación educativa en áreas tales como concepciones alternativas, diferencias entre expertos y novatos, adquisición de esquemas, sobrecarga cognitiva y análisis jerárquico, hemos desarrollado un marco para pensar sobre la organización del conocimiento y su uso para la comunicación, y sobre el razonamiento y la resolución de problemas. Basados en este marco y en nuestras reflexiones sobre el aprendizaje y la enseñanza, hemos creado una metodología didáctica llamada resolución de problemas basada en el análisis, diseñada para promover tanto la comprensión conceptual profunda como la capacidad de resolver problemas eficientemente a través de enfocarse en el análisis y el razonamiento como un puente entre las dos. Presentamos aquí una progresión de objetivos de enseñanza que lleva a los estudiantes de ser aprendices novatos a ser «resolvedores» de problemas más eficientes y más reflexivos. Para cada objetivo, resumimos los resultados relevantes de la investigación cognitiva y describimos las recomendaciones didácticas que se pueden derivar de ella. Luego hicimos la lista de algunas estrategias didácticas útiles para promover ese objetivo en particular y, finalmente, indicamos algunas prácticas de aula que deberían ayudar a que los estudiantes progresen desde esta perspectiva.
\end{abstract}

Palabras clave. Metodología didáctica basada en la investigación, comprensión conceptual, resolución de problemas, análisis y razonamiento, prácticas de aula.

Summary. Using the results of educational research coming from such areas as alternative conceptions, expert-novice differences, schema acquisition, cognitive overload, and hierarchical analysis, we have developed a framework for thinking about knowledge organization and its use for communication, reasoning, and problem solving. Based on this framework, as well as on our own reflections on learning and teaching, we have created an instructional approach called analysis-based problem solving that is designed to promote both deep, conceptual understanding and proficient problem-solving ability, by focusing on analysis and reasoning as a bridge between them both. We report here on a progression of instructional goals that takes students from novice learners to more proficient, more thoughtful problem solvers. For each goal, we summarize the relevant cognitive research results, and describe the pedagogy that can be derived from it. Then, we list some modes of instruction useful for promoting that particular goal, and finally, indicate some classroom practices that should help students make progress within the approach.

Keywords. Research-based instructional approach, conceptual understanding, problem solving, analysis and reasoning, classroom practices.

Si uno entra en muchas aulas actuales y observa la forma en que se enseña, pensará que el profesor está intentando verter el conocimiento en la cabeza de sus estudiantes, como se representa en la figura 1a. Los estudiantes escuchan pasivamente una clase magistral u observan una demostración, y puede que parezca que están absorbiendo todo lo que dice el profesor.

La figura 1b (adaptada de Van Heuvelen, 1992), de hecho, se acerca más a lo que está sucediendo realmente en muchas aulas. Los estudiantes también están pasivos, pero se retiene una parte muy pequeña del conocimiento. Por ejemplo, el profesor podría sorprenderse de que los estudiantes no entiendan algo después de que se les repite una o dos veces. O quizás los estudiantes obtengan resultados por debajo de las expectativas en cuestionarios, pruebas y exámenes. Esta representación es algo defectuosa, sin embargo, porque supone que hay comunicación eficiente en el aula y echa la culpa a los estudiantes de cualquier fallo en la comprensión. 

pero el conocimiento se derrama. c) El profesor trata de verter el conocimiento en la cabeza de la estudiante, pero el embudo invertido impide que buena parte del conocimiento entre. La estudiante está usando su dedo para intentar que el conocimiento no se derrame, pero no lo logra.

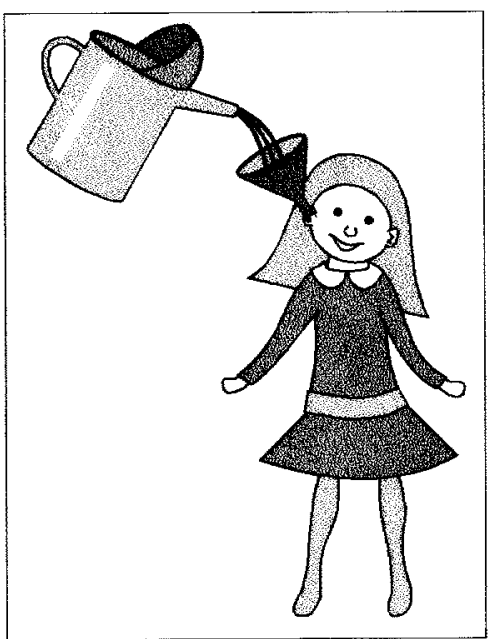

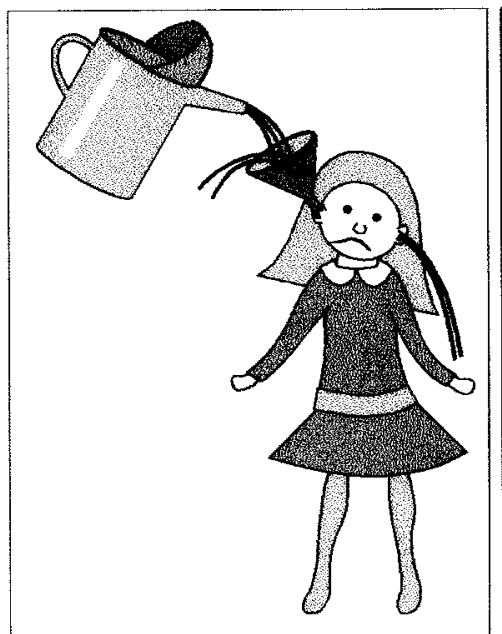

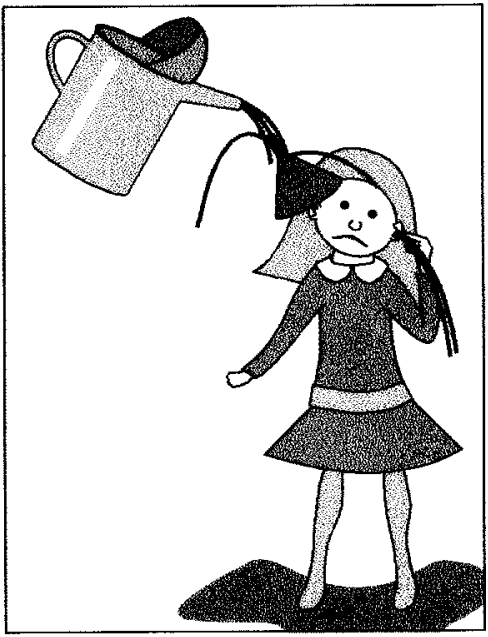

La figura 1c muestra nuestra visión de las prácticas didácticas comunes; hay dos rasgos para notar. Primero, el dedo en la oreja significa que los estudiantes están tratando de retener el conocimiento pero, al no poseer las habilidades necesarias, fracasan. Asistir a clases magistrales, tomar notas y estudiarlas, leer libros de texto, memorizar fórmulas, resolver problemas no es suficiente para aprender o entender el material deseado. Segundo, el embudo puesto al revés significa que las formas comunes de enseñanza crean un desencuentro entre los estudiantes y el profesor, lo cual puede llevar a que se produzca poca comunicación significativa. Los estudiantes no siempre entienden lo que dice el profesor, mientras que éste no recibe una retroalimentación acerca de lo que ellos entienden o no. La comunicación ocurre en una sola dirección -de los profesores a los estudiantes-, así que el profesor puede incluso no ser consciente de que hay confusión.

Aprender ciencias es particularmente difícil para muchos estudiantes. En el intento de cubrir los temas, muchos profesores se vuelcan en metodologías didácticas que son eficaces para transmitir (esto es, dar la clase y después poner muchos problemas para resolver como tarea) más que para aprender. Muchos profesores valoran la resolución de problemas y, generalmente, piensan que, si los estudiantes pueden resolver problemas, deben entender el material. La ironía de la enseñanza de la física es que, aunque los estudiantes resuelven muchos problemas, generalmente no desarrollan buenas habilidades de resolución. Resolver muchos problemas más bien favorece y refuerza aproximaciones que usan fór- mulas y un aprendizaje superficial. Además, el éxito en la resolución de problemas generalmente no es una buena medida de la comprensión conceptual.

Otros abordajes parecen sacrificar la resolución de problemas en favor de la comprensión profunda. Estas metodologías didácticas ponen tanta atención en los conceptos que la enseñanza y la evaluación de la comprensión conceptual se convierten en un objetivo más que en un medio para llegar a un fin. ¿Y cuáles son, pues, el valor y el propósito de la comprensión conceptual? Creemos que el valor de una comprensión profunda de los conceptos y los principios es ser capaz de aplicar flexiblemente el conocimiento para resolver problemas no familiares; por ello la capacidad de resolución de problemas sin comprensión conceptual no es valorada por la mayoría de los profesores.

Por lo tanto, un énfasis excesivo tanto en la resolución de problemas como en la comprensión conceptual no es deseable. Pero ambas son facetas valiosas de la enseñanza de la física. Entonces, ¿cómo se puede favorecer ambas sin ir demasiado lejos?

Creemos que tanto la comprensión profunda como la resolución de problemas eficiente surgen de ser capaces de analizar situaciones conceptualmente. Así, el análisis se vuelve el puente que permite que los conceptos sean útiles para la resolución de problemas. Nuestra aproximación, llamada resolución de problemas basada en el análisis, desplaza el peso de la enseñanza desde la clase magistral y la resolución de problemas, o desde las concep- 
ciones erróneas y la comprensión conceptual, hacia actividades que aspiran a procesos cognitivos beneficiosos.

Aprender ciencias es como subir una escalera. Para llegar arriba de todo, se necesita tener ambas piernas trabajando juntas. Una pierna nos sostiene mientras que la otra nos sube al siguiente escalón. De la misma manera, el análisis conceptual y la resolución de problemas trabajan juntos, apoyándose y mejorándose mutuamente.

Para ayudar a los estudiantes a desarrollar habilidades tanto de análisis conceptual como de resolución de problemas al mismo tiempo, los llevamos a través de una secuencia de experiencias de aprendizaje. Primero, los estudiantes exploran sus ideas previas para que éstas no interfieran con los conceptos científicos. Segundo, los estudiantes afinan, conectan e interrelacionan los conceptos, creando una rica red de ideas que les ayuda a comprenderlos y recordarlos. Tercero, los estudiantes aprenden cómo usar los conceptos para analizar y razonar sobre situaciones comunes, lo que les hace posible resolver problemas interesantes, inusuales o complejos. Cuarto, los estudiantes desarrollan habilidades generales de resolución de problemas basadas más en estrategias similares a las de los expertos - que usan principiosque en aproximaciones como las de los novatos -que usan rasgos superficiales. Quinto, los estudiantes organizan y priorizan su propio conocimiento a fin de que sea particularmente útil para la resolución de problemas y para el análisis.

Una consecuencia de esta perspectiva es que los estudiantes pueden resolver problemas más difíciles que otros estudiantes que siguen el enfoque tradicional, y generalmente mejoran sus habilidades de razonamiento y desarrollan una comprensión más profunda de los conceptos y principios. Los conceptos, más que las ecuaciones, se convierten en el lenguaje que los estudiantes usan para aprender y para hacer física.

Para cada uno de los cinco tipos de experiencias de aprendizaje descritas en el párrafo anterior, presentaremos resultados de investigación específicos y fundamentos didácticos que son relevantes para comprenderlas. Luego recomendaremos algunas estrategias didácticas y prácticas de aula que creemos que son particularmente útiles a fin de crear experiencias educativas ricas para los estudiantes. Estas estrategias y prácticas han sido usadas para crear un extenso conjunto de materiales instruccionales de física (Leonard et al., 1999, 2000, 2001).

\section{EXPLORAR LOS CONCEPTOS PREEXIS- TENTES DE LOS ESTUDIANTES}

\section{Resultados de la investigación}

Los estudiantes llegan a la clase con ideas profundamente arraigadas acerca de cómo funciona el mundo, muchas de las cuales sobreviven a pesar de la enseñanza y a menudo coexisten con las ideas «científicas». Estas concepciones alternativas en general surgen porque la mente humana trata constantemente de dar cuenta y hacer frente a las experiencias. De esta manera, los estudiantes ya tienen un marco conceptual cuando comienzan un curso de ciencias, aunque este marco pueda parecer inconsistente para un experto o un profesor.

Se ha mostrado que las concepciones previas en muchos casos impiden el aprendizaje de marcos más formales. Las concepciones previas pueden ser difíciles de erradicar y a menudo se mantienen incluso después de que los profesores han hecho un esfuerzo conjunto para deshacerse de ellas (para revisiones y bibliografía, ver McDermott, 1984; Mestre, 1991, 1994; Pfundt y Duit, 1991; Maloney, 1992; McDermott y Redish, 1999).

La investigación también indica que el esquema organizacional de cada persona es único y que la gente a menudo tiene dificultades para usar o comprender plenamente el esquema de otra persona. Los mejores estudiantes a menudo buscan patrones propios, pero la mayor parte de los estudiantes necesita tener patrones que le sean explícitamente indicados (Gick y Holyoak, 1980, 1983, 1987; Bassok y Holyoak, 1989).

Observaciones de clase en todos los niveles indican que los profesores rara vez tienen en cuenta el conocimiento conceptual previamente construido por los estudiantes. Además, las ideas, predicciones y explicaciones de los fenómenos científicos que tienen los estudiantes no son examinadas para determinar si los conceptos que se enseñan están en conflicto con sus ideas previas (Resnick, 1983; Hewson et al., 1995). Los resultados de investigación sugieren fuertemente otras dos cuestiones: a) que los profesores generalmente no están al tanto del estado de conocimiento de sus estudiantes; y $b$ ) que las prácticas de evaluación tradicionales no miden la comprensión conceptual (Chi y Glaser, 1981; Kulm y Stuessy, 1991; Harmon y Mungal, 1992; Hestenes y Wells, 1992; Hestenes et al., 1992).

\section{Fundamentos didácticos}

El constructivismo es una filosofía que simplemente afirma que todo el conocimiento es construido como resultado de procesos cognitivos dentro de la mente humana. Rechaza la idea de que el conocimiento sea la representación de una realidad externa independiente del observador. (La ciencia, por supuesto, presupone la existencia de una realidad externa y busca describir y explicar su naturaleza y su comportamiento.)

\section{Las premisas del constructivismo son:}

- El conocimiento es construido, no transmitido. Las experiencias deben ser interpretadas y procesadas por cada individuo. Dos personas no pueden intercambiar conocimiento como si fuera sólo información.

- El conocimiento previo tiene impacto en el aprendizaje. Los marcos cognitivos preexistentes determinan a 
qué presta atención la gente, cómo interpreta aquello a lo que presta atención y cómo construye nuevo conocimiento. Dos personas pueden tener la misma experiencia pero interpretarla de distinta manera.

- La comprensión inicial es local, no global. Las nuevas ideas son necesariamente introducidas y entendidas sólo en un contexto limitado. Cuando se introduce una idea por primera vez, puede ser difícil para alguien saber qué rasgos de la situación son más relevantes para entenderla. Después, cuando la idea ha sido explorada en una variedad de contextos, resulta generalmente más fácil percibir el patrón presupuesto, y la comprensión es generalmente más amplia.

- Construir estructuras útiles de conocimiento requiere una actividad esforzada e intencionada. El aprendizaje significativo requiere una participación activa y reflexiva.

Con fines didácticos, las premisas del constructivismo pueden ser reformuladas como sigue:

- Los estudiantes tienen una visión del mundo establecida, formada con los años de experiencias previas y aprendizaje.

- Incluso, a medida que evoluciona, la visión del mundo de un estudiante filtra todas las experiencias y afecta a todas las interpretaciones de posteriores observaciones.

- Los estudiantes están emocionalmente vinculados a su visión del mundo y no la abandonan fácilmente.

- Cuestionar, revisar y reestructurar la propia visión del mundo requiere mucho esfuerzo.

El primer paso en el proceso de aprendizaje, por tanto, es hacer que los profesores y los estudiantes sean conscientes de su visión del mundo. Cuanto más saben los profesores acerca de los marcos conceptuales individuales de los estudiantes, mejor pueden poner en evidencia las limitaciones de dichos marcos, y más probable es que puedan inducir a los estudiantes a repensar y reformular su propia visión del mundo (Resnick, 1983, 1987; Anderson, 1987; Di Sessa, 1988; Von Glasersfeld, 1989, 1992; Schauble, 1990; Ritchie et al., 1997). Los estudiantes deberían autorregularse y participar plenamente en el proceso de aprendizaje. Los estudiantes pueden buscar deliberadamente experiencias de aprendizaje suplementarias y pueden ser muy efectivos a la hora de modificar sus propias visiones del mundo (Brown y Clement, 1989; Clement, 1993; Camp y Clement, 1994; Mestre et al., 1997; Wenk et al., 1997).

\section{Estrategias didácticas}

Algunas maneras útiles de explorar los conceptos preexistentes de los estudiantes son:

- Usar el «predecir-y-mostrar» (inadecuación del modelo antiguo). Cuando los estudiantes predicen el resul- tado de una demostración o un experimento, están más comprometidos con la actividad y es menos probable que no expongan sus ideas, esto es, menos probable que esperen a que el profesor haga una interpretación por ellos. Prediciendo un resultado, los estudiantes a menudo ponen de manifiesto las características de la situación que analizan y muestran cuáles de ellas consideran más relevantes para comprenderla. Aunque a menudo la visión del mundo de cada estudiante permanece implícita durante esta estrategia didáctica, una predicción incorrecta puede ser ideal para demostrar que su modelo tiene una aplicabilidad limitada y a prepararlo para aprender.

- Explicar (dibujar, describir, discutir). Cuando los estudiantes explican su razonamiento para una respuesta, dibujan una imagen de algo, describen una observación o discuten una demostración, se ven forzados a usar y explicitar los modelos que tienen para organizar sus experiencias, y a utilizar conceptos en sus explicaciones o descripciones. Esta estrategia didáctica promueve la autorregulación; los estudiantes no pueden cambiar su visión del mundo eficazmente a menos que sean conscientes de ella.

- Comunicar acerca del proceso de aprendizaje. Los estudiantes necesitan saber que cada uno de ellos tiene una perspectiva única, y que a veces ésta no es autoconsistente y puede impedirles aprender eficientemente y llegar a una comprensión profunda. Los estudiantes necesitan volverse participantes activos durante toda la experiencia educativa, desde la exploración de los conceptos preexistentes hasta la estructuración del conocimiento. La comunicación sobre el aprendizaje es una forma de ayudarlos.

\section{Prácticas de aula}

Hay muchas maneras en las que los profesores pueden ayudar a los estudiantes a manifestar y confrontar su visión del mundo; entre otras:

- Hacer que los estudiantes usen sus propios modelos para responder preguntas abiertas. Las buenas preguntas son a menudo preguntas simples en un contexto familiar; ponen de manifiesto las limitaciones de los modelos de los estudiantes y los motivan a buscar nuevas concepciones. Las respuestas dan información a los profesores y promueven la autorregulación en los estudiantes.

- Decir a los estudiantes que no deben buscar respuestas «correctas». Las respuestas deberían ser aquello que cada estudiante cree que es verdadero. La parte difícil es crear una atmósfera de aula en la cual los estudiantes estén cómodos arriesgándose y exponiendo sus propias ideas, más que sintiéndose forzados a dar a los profesores lo que ellos quieren. En un ambiente constructivista, es más probable que los estudiantes se involucren en el proceso de aprendizaje y comprensión. Cuando las preguntas involucran experiencias comunes, las respuestas pueden a menudo ser contrastadas con 
Tabla I

Una comparación entre las características del conocimiento de los expertos y el de los novatos.

\begin{tabular}{|l|l|}
\hline \multicolumn{1}{|c|}{ Experto } & \multicolumn{1}{c|}{ Novato } \\
\hline$\cdot$ Grandes cantidades de conocimiento específico de dominio & $\bullet$ Conocimiento desorganizado \\
\hline$\cdot$ Conocimiento ricamente interconectado & $\bullet$ Conocimiento mayormente desconectado y amorfo \\
\hline$\cdot$ Conocimiento jerárquicamente estructurado & $\bullet$ Conocimiento almacenado cronológicamente \\
\hline - Múltiples representaciones integradas & $\bullet$ Representaciones pobremente formadas y sin relaciones \\
\hline$\cdot$ Recuerdo bueno & $\bullet$ Recuerdo pobre \\
\hline
\end{tabular}

simples experimentos o demostraciones, y la ciencia se integra a la vida diaria de los estudiantes.

- Usar pequeños grupos. No es necesario que el profesor confronte la visión del mundo de todos los estudiantes; otro estudiante puede ser tan efectivo como el profesor -a veces incluso más- para descubrir inconsistencias en una visión del mundo. Las discusiones en pequeños grupos ayudan a los estudiantes a refinar sus explicaciones sobre el razonamiento usado para responder preguntas, y son muchas veces menos amenazadoras que las discusiones en plenario. Usar pequeños grupos hace que los estudiantes tomen conciencia de diversas perspectivas y promueve la idea de que lo que todos piensan es importante. También ayuda a los estudiantes a afinar sus definiciones; y lo que es quizás más impor- tante: usar pequeños grupos integra el lenguaje con la ciencia y la experiencia.

\section{AFINAR Y AGRUPAR CONCEPTOS}

\section{Resultados de investigación}

La tabla I resume las principales diferencias entre las características del conocimiento de expertos y novatos (Larkin, 1979; Chi y Glaser, 1981; Glaser, 1992).

\section{Fundamentación didáctica}

Hemos organizado estos resultados en una representación del bagaje de conocimiento del experto, como se muestra en la figura 2 .

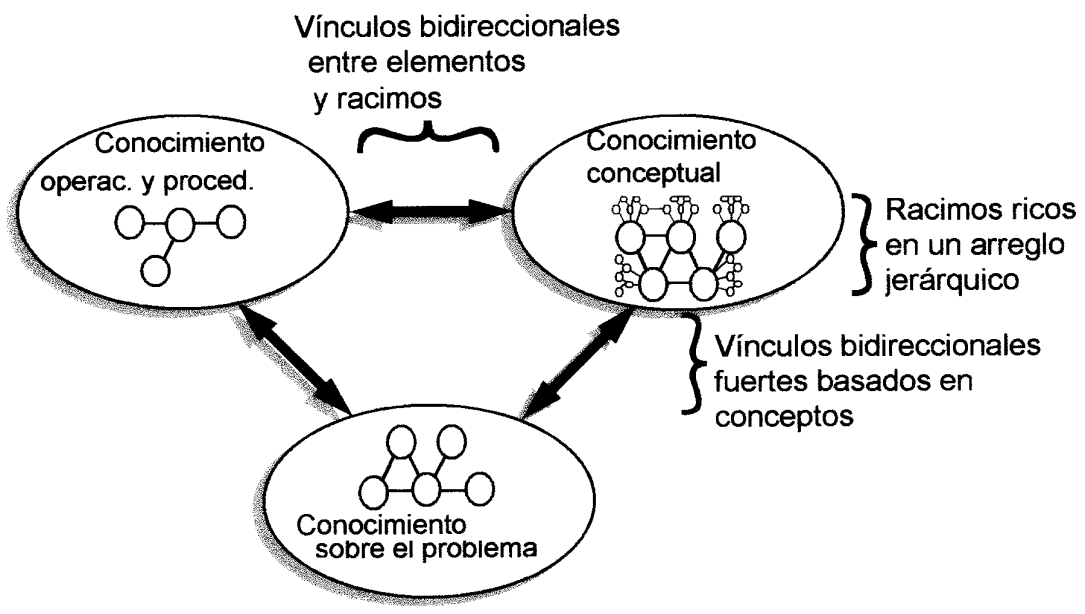


Figura 3

Una representación del almacén de conocimiento del novato.

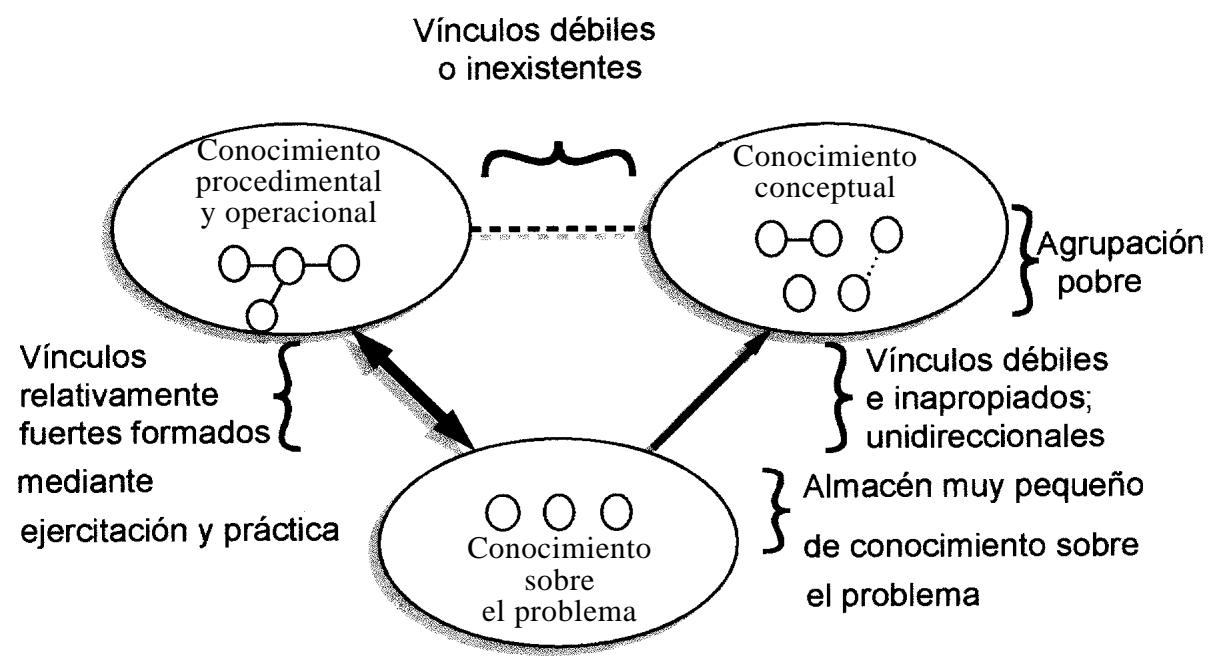

El conocimiento disciplinar se ha dividido en tres categorías generales:

- Conocimiento operacional y procedimental, como la definición de la energía cinética, cómo dibujar un diagrama de cuerpo libre y cómo hallar la fuerza normal.

- Conocimiento sobre el problema, esto es, los rasgos del problema o situación usados para caracterizarlo. Estos rasgos pueden ser rasgos superficiales, tales como un plano inclinado o un resorte, o conceptos y principios, tales como que la energía se conserva.

- Conocimiento conceptual, como fuerza, masa, aceleración, energía cinética, etc.

El experto tiene una agrupación rica y jerárquica (priorizada) del conocimiento conceptual. Los conceptos están conectados a muchas operaciones, procedimientos y situaciones problemáticas diferentes, refinando así los significados de los conceptos. Los expertos usan los conceptos para caracterizar las situaciones problemáticas y también para juzgar la pertinencia y la aplicabilidad de las ecuaciones, las operaciones y los procedimientos.

El novato tiene una estructura de conocimiento muy diferente, como se muestra en la figura 3. A diferencia de los expertos, los novatos generalmente tienen una agrupación pobre de los conceptos. Muchos de los vínculos son inadecuados; otros no existen. Algunos de los vínculos inapropiados son extremadamente fuertes, lo que puede llevar a concepciones erróneas. Muchos novatos están familiarizados o han memorizado un gran número de ecuaciones, pero a menudo las recuerdan incorrectamente o necesitan buscarlas para usarlas. Se les han enseñado operaciones y procedimientos, pero no son aún eficaces usándolos y, por lo tanto, los evitan. Los vínculos entre las ecuaciones y las situaciones problemáticas son relativamente fuertes, pero están mayormente basados en las cantidades (datos e incógnitas) mencionadas en el problema o la situación.

Según nuestro punto de vista, un objetivo de la enseñanza debería ser ayudar a los estudiantes a desarrollar una estructura de conocimiento rica basada en conceptos. Para hacer esto, los estudiantes deben aprender a afinar (afilar) y agrupar (interrelacionar) ideas (Minstrell, 1992).

\section{Estrategias didácticas}

Aquí hay algunas estrategias relevantes para ayudar a los estudiantes a afinar y agrupar conceptos:

- Usar representaciones múltiples. Una representación puede ser lingüística, abstracta, verbal, simbólica, experiencial, pictórica, física o gráfica. La comprensión profunda de cualquier concepto requiere muchas representaciones; aun así, los estudiantes muchas veces creen que una representación (la algebraica) es suficiente. Los estudiantes tampoco tienden a interrelacionar representaciones, lo que a menudo significa que sus ideas físicas abstractas no están bien conectadas a experiencias del mundo real. Usar diferentes representaciones para el mismo conocimiento y hacer que los estudiantes traduzcan entre representaciones los ayuda a interrelacionar ideas y a relacionar las ideas con la experiencia personal; 
por ejemplo, escribir una ecuación en la pizarra y pedir a los estudiantes que la lean (esto es, que traduzcan de la representación algebraica a la verbal). Nos gusta especialmente usar gráficas porque son abstractas, como las ecuaciones, pero pueden ser entendidas cualitativamente, como los diagramas o las figuras.

- Explorar contextos extendidos. La comprensión inicial está necesariamente limitada por el contexto en el cual es introducida por primera vez. La mente humana busca naturalmente patrones y tiende a generalizar usando los rasgos más notables. Los estudiantes tienden a concentrarse en rasgos superficiales y a menudo generalizan incorrectamente como resultado. Tampoco pueden reevaluar fácilmente sus generalizaciones. Investigar un conjunto amplio de situaciones problemáticas ayuda a los estudiantes a afinar y abstraer conceptos; evita generalizaciones inapropiadas o sobresimplificadas. Es más probable que los estudiantes usen los rasgos relevantes e ignoren los irrelevantes después de que han explorado una variedad de contextos.

- Usar "comparar-y-contrastar». Como en el caso de los contextos extendidos, el objetivo de comparar y contrastar es la interrelación del conocimiento. La diferencia es que, mientras comparan y contrastan situaciones, los estudiantes necesitan atender explícitamente a las semejanzas y diferencias entre ellas.

- Explicar (describir, discutir, definir). Cuando los estudiantes explican (describen, etc.) su razonamiento, ponen de manifiesto los rasgos que están usando para organizar sus ideas. Explicar también ayuda a los estudiantes a crear conexiones entre ideas.

\section{Prácticas de aula}

El objetivo de estas actividades es ayudar a los estudiantes a afinar y generalizar a definiciones de los conceptos, y a relacionar nuevas ideas con las ya aprendidas. Las siguientes prácticas de aula están orientadas a conseguir este objetivo.

- Usar tantas representaciones diferentes como sea posible para un mismo concepto. Usar diferentes representaciones ayuda a los estudiantes a afinar su comprensión y provee alternativas para pensar sobre un concepto. Esto puede ser especialmente importante cuando un concepto en particular es necesario para comprender otro concepto más complejo.

- Asegurarse de que los primeros ejemplos de algo sean iguales sólo en el rasgo (o rasgos) que son relevantes para entenderlos. Es probable que los estudiantes noten muchas semejanzas entre dos situaciones. No podemos garantizar que notarán lo que es relevante e ignorarán lo que no lo es. ¡Decirles a qué prestar atención y qué ignorar no es suficiente! Por ejemplo, muchos estudiantes creen que la fuerza normal siempre apunta verticalmente hacia arriba, porque todos los ejemplos que han visto comparten este rasgo. Teniendo más cuidado al elegir los primeros dos o tres ejemplos de algo, los profesores pueden ayudar a sus estudiantes a evitar las generalizaciones sobresimplificadas y la confusión.

- Hacer preguntas que exploren los límites del conocimiento de los estudiantes. El aprendizaje se produce desde un núcleo de ideas bien formadas hacia el exterior y la comprensión tiene lugar en la periferia cuando los estudiantes intentan usar y relacionar ideas parcialmente formadas. El aprendizaje no puede tener lugar en la oscuridad y confusión de ideas pobremente formadas. Por ejemplo, haga que los estudiantes comparen situaciones similares: ¿Es [algo] lo mismo o diferente? ¿Por qué? Cambie algo sobre la situación; ¿qué más cambia? Haga que los estudiantes proporcionen ejemplos (preferiblemente de sus experiencias de la vida cotidiana) en los cuales el concepto se pone de manifiesto.

\section{DESARROLLAR HABILIDADES DE ANÁLI- SIS Y DE RAZONAMIENTO}

\section{Resultados de la investigación}

La mayor parte de los estudiantes de física que comienzan no aprecian el valor de un análisis conceptual como parte de la resolución de problemas. En cambio, los novatos usualmente realizan un análisis «medios-fines». Se concentran en las ecuaciones y comienzan a manipularlas en un intento de aislar las incógnitas buscadas, a menudo insertando valores numéricos desde el inicio del proceso. Los novatos se distraen en el intento de determinar el valor de la cantidad deseada. En este estado mental, los estudiantes a menudo sufren una sobrecarga cognitiva. Están tan concentrados en las respuestas que no les quedan recursos mentales libres para pensar en la resolución de problemas (Dufresne et al., 1992; Larkin, 1981, 1983).

\section{Fundamentación didáctica}

La manera en que los estudiantes aprenden es en sí misma aprendida. Lo que los estudiantes saben determina cómo participan en las actividades de resolución de problemas, y esta participación a su vez determina lo que aprenden. El abordaje de los estudiantes ha sido reforzado por años de aprendizaje ritualizado, memorización y exposición. Continuar dando grandes cantidades de problemas contribuye poco a romper este ciclo. De hecho, resolver problemas tradicionales puede reforzar actitudes superficiales y disuadir a los estudiantes de querer comprender (Brown et al., 1989; Touger et al., 1995).

Una solución es estructurar actividades de resolución de problemas para desviar la atención de los estudiantes de la obtención de una respuesta y para comunicarse con ellos sobre el tema del aprendizaje, para que busquen patrones y explicaciones alternativas. Por ejemplo, se debería hacer conscientes a los estudiantes de sus hábitos de aprendizaje. Antes de que los estudiantes puedan usar los conceptos para resolver problemas, deben aprender 
cómo usar esos conceptos para analizar situaciones y razonar sobre ellas (Dufresne et al., 1997).

\section{Estrategias didácticas}

Algunas estrategias útiles para desarrollar habilidades de análisis y razonamiento son:

- Usar múltiples representaciones. La esencia del razonamiento eficaz es encontrar la representación en la cual el resultado es más obvio. (Por ejemplo, en muchas situaciones, los diagramas de cuerpo libre son útiles para comparar las magnitudes de las fuerzas. También una gráfica puede ser útil para razonar una respuesta.) Cuando los estudiantes consideran diferentes representaciones y las utilizan para analizar situaciones, pueden mejorar sus habilidades de pensamiento crítico; esto puede llevar a una mejor capacidad en la de resolución de problemas.

- Usar «comparar-y-contrastar». A menudo toma menos tiempo comparar dos cantidades o situaciones que calcular una sola cantidad. Muchas veces podemos describir una situación sin especificar información suficiente para determinar las incógnitas, pero con suficiente información para hacer una comparación. (Por ejemplo, considérense un disco y una esfera con la misma masa y el mismo radio rodando a la misma velocidad a lo largo de una superficie horizontal. Al llegar a un plano inclinado, ¿cuál de los dos alcanzará una altura máxima más grande?)

- Explicar (resumir, discutir, escuchar, debatir, argumentar). Las buenas habilidades de pensamiento crítico pueden ser desarrolladas y afinadas haciendo que los estudiantes expliquen sus razonamientos y debatan sobre cuestiones. Escuchar requiere análisis y procesamiento. Presentar contraargumentos a la línea de razonamiento de alguien densifica su estructura de conocimiento. Razonar necesariamente involucra conceptos; éstos se vuelven el vocabulario de las explicaciones. El análisis cualitativo se convierte así en una herramienta viable para la resolución de problemas.

\section{Prácticas de aula}

Las siguientes sugerencias pueden ayudar a los estudiantes a enfocar su atención en la utilidad de los conceptos para comprender situaciones físicas:

- Usar actividades sin objetivos. Para reducir la carga cognitiva y fomentar la reflexión y el pensamiento profundo, los estudiantes necesitan trabajar sobre preguntas que no requieran un resultado numérico. Esto hace más probable que usen conceptos. Haga preguntas que sólo puedan ser contestadas usando conceptos o que al menos puedan ser contestadas más fácilmente usándolos. Se pueden usar ecuaciones, pero usualmente éstas no se manipularán para encontrar una incógnita. Por ejemplo, podría usarse una definición para determinar cómo se relaciona una cantidad con otra.

- Dirigir la atención de los estudiantes a los rasgos de una situación más relevante para comprenderla. Por ejemplo, en una situación, la energía podría ser lo más relevante para entenderla, mientras que, en otra similar, la cantidad de movimiento podría ser más relevante. Los

Tabla II

Una comparación entre las conductas de resolución de problemas de los expertos y los novatos.

\begin{tabular}{|l|l|}
\hline \multicolumn{1}{|c|}{ Experto } & Novato \\
\hline $\begin{array}{l}\text { - El conocimiento conceptual influye en la resolución } \\
\text { de problemas. }\end{array}$ & $\begin{array}{l}\text { - La resolución de problemas es altamente independiente } \\
\text { de los conceptos. }\end{array}$ \\
\hline $\begin{array}{l}\text { A menudo realiza un análisis cualitativo, especialmente } \\
\text { cuando está atascado. }\end{array}$ & $\bullet$ Usualmente manipula ecuaciones. \\
\hline - Usa estrategias basadas en conceptos que anticipan la acción. & $\begin{array}{l}\text { - Usa técnicas «medios-fines» que se orientan a corregir } \\
\text { disfunciones. }\end{array}$ \\
\hline - Tiene una variedad de métodos para salir de un atasco. & $\bullet$ Generalmente no puede salir de un atasco sin ayuda externa. \\
\hline - Puede pensar acerca de la resolución de problemas mientras \\
$\begin{array}{l}\text { los resuelve. } \\
\text { - Es capaz de verificar una respuesta usando } \\
\text { un método alternativo. }\end{array}$ & $\bullet \begin{array}{l}\text { En la resolución de problemas usa todos los recursos } \\
\text { mentales disponibles. }\end{array}$ \\
\hline
\end{tabular}


estudiantes podrían centrarse en las semejanzas superficiales y no darse cuenta de que se utilizan diferentes conceptos. Analizar situaciones fomenta el uso de conceptos y principios científicos para organizar el conocimiento.

- Usar situaciones familiares o simples o usar la misma situación para hacer muchos tipos diferentes de preguntas. Los estudiantes invierten muchos recursos mentales en procesar y almacenar el contexto en el cual se formula una pregunta. Cuando la situación es poco familiar o complicada, pueden quedar pocos recursos para que los estudiantes la analicen. Usando situaciones familiares o relativamente simples, se reduce la carga cognitiva. Reutilizando las situaciones en muchos problemas y cuestiones diferentes, los estudiantes aprenden que la misma situación pueden ser analizada y comprendida usando muchos conceptos y principios diferentes, y que los rasgos superficiales no siempre son útiles para organizar el conocimiento.

\section{DESARROLLAR HABILIDADES DE RESO- LUCION DE PROBLEMAS}

\section{Resultados de la investigación}

El comportamiento de los expertos y los novatos en la resolución de problemas es muy diferente, como se resume en la tabla II (Larkin et al., 1980a, 1980b; Chi et al., 1981; Hardiman et al., 1989; Glaser, 1992).

\section{Fundamentación didáctica}

La figura 4 muestra una representación del conocimiento estratégico. El conocimiento estratégico conecta el conocimiento de las situaciones problemáticas, las ecuaciones, las operaciones y los procedimientos con un elemento estratégico que guía todo el proceso de resolución de problemas. Con base en un análisis conceptual, se toman decisiones con respecto a qué ideas se han de tener en cuenta y cuáles han de ser ignoradas. Los estudiantes no construirán por sí mismos elementos de conocimiento estratégico. Deben participar en actividades estructuradas de resolución de problemas y deben reflexionar sobre el proceso de resolución (Dufresne et al., 1992).

La resolución de problemas eficiente consiste principalmente en realizar elecciones -en qué concentrarse, qué principio aplicar, qué representación usar, qué ignorar. Sin embargo, la mayoría de los estudiantes tiene, como máximo, una manera de resolver cada problema en particular. Las elecciones necesariamente involucran conceptos, porque son los conceptos los que se usan para hacer comparaciones. Cuanto más fomentemos que los estudiantes hagan análisis cualitativos antes de resolver los problemas, más mejorarán su habilidad para resolverlos.

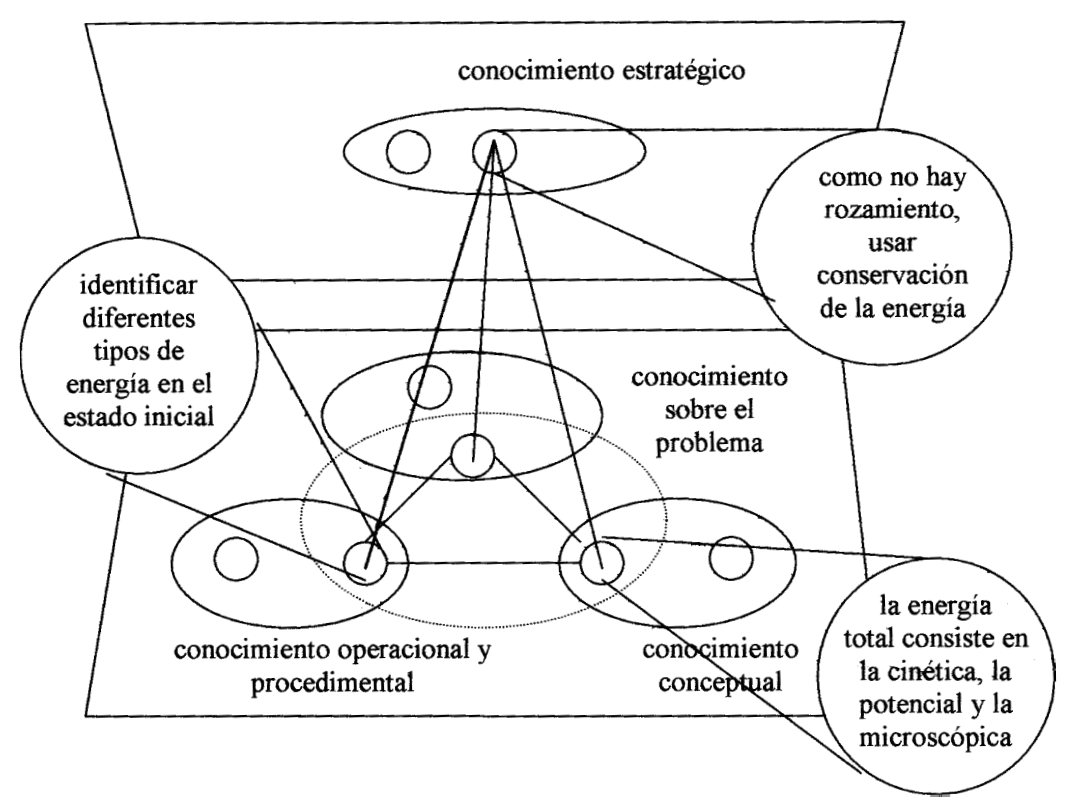




\section{Estrategias didácticas}

Las siguientes estrategias pueden usarse para ayudar a los estudiantes a desarrollar abordajes positivos para la resolución de problemas y a abandonar abordajes tradicionales del tipo «medios-fines»:

- Usar el «clasificar-y-categorizar». Este método es similar a «comparar-y-contrastar», excepto que el foco aquí está en clasificar ideas o problemas y en dar nombre a las categorías resultantes. Los estudiantes deben ejercitarse en la creación y el reconocimiento de sistemas de clasificación. Cuando los estudiantes clasifican, eligen nombres para sus categorías y explican sus sistemas, aumenta la probabilidad de que usen conceptos para organizar su conocimiento.

- Generar soluciones múltiples. Cuando los estudiantes resuelven el mismo problema usando diferentes abordajes, aprenden a priorizarlos. Por ejemplo, la representación algebraica no siempre es la más útil para resolver un problema, pero los estudiantes no están generalmente convencidos de eso.

- Planificar, justificar y crear estrategias. Muy pocas relaciones en física son válidas siempre. Muchas están definidas o se derivan para un conjunto particular de circunstancias. (Por ejemplo, la definición de la energía cinética como $1 /{ }_{2} \mathrm{mv}^{2}$ sólo es válida para un objeto puntual o cuando se trata de un cuerpo rígido que no está rotando.) Muchos estudiantes no son conscientes de las suposiciones, condiciones y circunstancias que hacen aplicable una particular ecuación, operación o procedimiento. Haciendo que los estudiantes planifiquen su abordaje (sin resolver realmente el problema), lo justifiquen o desarrollen una estrategia, se conseigue que aprendan el valor de los conceptos y del análisis conceptual para la resolución de problemas (Leonard et al., 1996).

\section{Prácticas de aula}

Aquí se exponen algunas ideas para ayudar a los profesores a implementar actividades estructuradas de resolución de problemas:

- Elegir problemas que requieran un análisis conceptual para ser resueltos. Los problemas tradicionales son usualmente resueltos aplicando una o más ecuaciones previamente derivadas o repitiendo de memoria procedimientos estándar. No es necesario entender para resolver los problemas tradicionales. Sin embargo, cuando los problemas no pueden ser resueltos usando una aproximación con fórmulas, es más probable que los estudiantes se den cuenta de que el método más eficaz es usar conceptos. Esto no significa que los problemas tengan que ser difíciles (aunque los estudiantes los encontrarán difíciles al principio). De hecho, los mejores problemas usan situaciones simples of familiares y son relativamente fáciles de resolver con una abordaje basado en el análisis.
- Hacer que los estudiantes expliquen cómo resolverían un problema. En el tiempo que lleva a muchos estudiantes resolver un único problema, pueden explicar cómo resolverían varios. Esta actividad muestra a los estudiantes ( ¡y al profesor!) en qué se están enfocando cuando resuelven problemas. Ello influye en las discusiones posteriores y permite al profesor planificar intervenciones adecuadas.

- Hacer que los estudiantes resuelvan el mismo problema usando diferentes abordajes. Por ejemplo, muchos problemas pueden ser resueltos usando las leyes de Newton o la conservación de la cantidad de movimiento. Resolver el mismo problema por segunda vez usando un principio diferente ayuda a los estudiantes a aprender material nuevo y promueve la comparación entre métodos de resolución de problemas.

\section{ESTRUCTURAR EL CONOCIMIENTO EN LA MEMORIA}

\section{Resultados de la investigación}

En una serie de estudios, los estudiantes resolvían problemas contestando a una secuencia de preguntas organizada jerárquicamente. Por ejemplo, se les preguntaba primero: «¿Cuál de las siguientes propuestas usarías para resolver este problema? (cinemática / leyes de Newton; trabajo-energía / conservación de la energía; impulso-cantidad de movimiento / conservación de la cantidad de movimiento; o impulso angular - cantidad de movimiento angular / conservación de la cantidad de movimiento angular)». Dependiendo de sus respuestas a esta primera pregunta, se les pedía que respondieran preguntas cada vez más específicas sobre el problema. Los grupos control resolvían los mismos problemas en el mismo tiempo que el grupo bajo prueba.

De estos estudios emergieron dos resultados relevantes: a) era más probable que los estudiantes obligados a resolver problemas jerárquicamente lo hicieran con corrección; y $b$ ) era más probable que estos estudiantes clasificaran los problemas de acuerdo con el principio que se utilizaba para resolverlos (Dufresne et al., 1992; Mestre et al., 1993).

\section{Fundamentación didáctica}

Las tareas tradicionales de resolución de problemas no ayudan a los estudiantes a desarrollar habilidades útiles de resolución. Creemos que la razón es que los problemas tradicionales no estimulan procesos cognitivos beneficiosos. El núcleo de nuestro marco cognitivo es el siguiente:

- Se necesitan tipos particulares de conocimiento y de estructuras de conocimiento para una resolución de problemas eficiente y una comprensión profunda. 
- Son necesarios tipos particulares de procesos cognitivos para la adquisición de conocimiento conceptual y la construcción de estructuras de conocimiento útiles.

- Se deberían diseñar las actividades para dar lugar a los procesos cognitivos deseables.

El fin último sigue siendo la eficiencia en la resolución de problemas, pero objetivos igualmente importantes son desarrollar una comprensión profunda de las situaciones físicas y la capacidad de analizar situaciones nuevas o no familiares usando principios físicos. Para alcanzar estos objetivos, el foco principal de muchas actividades se desplaza de la resolución de problemas a la cognición. No podemos garantizar que ocurrirán procesos cognitivos beneficiosos, pero podemos hacer que aumente la probabilidad de que ocurran. Esto debería ser, creemos, el foco de la enseñanza (Leonard y Gerace, 1996; Dufresne et al., 1997).

\section{Estrategias didácticas}

Estas estrategias pueden ayudar a los estudiantes a estructurar su conocimiento para una comprensión profunda y para una resolución de problemas eficiente y efectiva:

- Hacer referencias hacia delante y hacia atrás. Los conceptos requieren mucho tiempo y experiencia para formarse completamente. No se puede esperar que los estudiantes aprendan completamente un tema o una idea antes de seguir adelante. Haciendo referencias hacia delante respecto al material que vendrá después, se prepara al estudiante. Mediante referencias hacia atrás, se asocia el nuevo material con material total o parcialmente establecido, consiguiendo un conocimiento entrelazado e interconectado, más que lineal o cronológico. Haciendo referencias hacia delante y hacia atrás, los estudiantes pueden construir muchos caminos hacia las mismas ideas; de esta manera, logran que el conocimiento sea más fácilmente accesible para la discusión, el análisis, el razonamiento y la resolución de problemas. Los estudiantes pueden también mejorar su desempeño en exámenes, tests y proyectos.

- Usar el «clasificar-y-categorizar». Cuando los estudiantes clasifican y categorizan las ideas, podemos aumentar la sofisticación de las ideas usadas para pensar sobre la física y las situaciones físicas. En particular, es más probable que los estudiantes usen principios y leyes físicas para clasificar los problemas y las situaciones problemáticas. La cuestión es: «¿son sus estudiantes conscientes de las categorías que usted cree que son útiles para organizar las ideas físicas?». Si es así, entonces ellos deberían ser capaces de darse cuenta cuando dos problemas son resueltos usando el mismo principio, incluso si los problemas parecen muy diferentes. Si no es así, la clasificación y la categorización pueden mostrar cómo los estudiantes organizan los problemas, y pueden conducir a discusiones útiles sobre otros posibles sistemas de organización.
- Reflexionar (evaluar, integrar, extender, generalizar, etc.). Después de muchas de las actividades, los estudiantes sacan beneficio de reflexionar sobre lo que acaban de hacer. ¿Qué patrones han percibido los estudiantes en las preguntas, situaciones o problemas presentados? ¿Cómo afrontarán situaciones similares en el futuro? ¿Qué dificultades han experimentado? ¿Qué es lo que causó esas dificultades? ¿Cómo las superarán en el futuro? ¿Cómo aplicarían las ideas [a una situación especificada]? ¿Pueden conectar esas ideas a eventos y situaciones del «mundo real»? ¿Qué principios generales pueden extraerse de la experiencia de aprendizaje? ¿Qué han aprendido los estudiantes?

Las experiencias de aprendizaje a menudo dan a los estudiantes las piezas necesarias del rompecabezas de «cómo-hacer-física», pero muchos estudiantes no intentarán juntar las piezas a menos que se les requiera específicamente y se les dé tiempo para hacerlo. La reflexión ayuda a los estudiantes a estructurar su conocimiento a medida que lo van aprendiendo.

- Comunicar acerca del proceso de aprendizaje. Para aprender física (¡y muchas otras asignaturas!), los estudiantes deben ser responsables del proceso de aprendizaje; deben ser más conscientes y estar más motivados; deben saber por qué aprender física es útil e importante. Se tienen en cuenta estos asuntos intentando dar información sobre el aprendizaje. ¿Saben los estudiantes cómo aprenden mejor? ¿Han pensado alguna vez cuáles son sus mayores fortalezas (o debilidades)? ¿Saben cuál es el propósito de una actividad en particular? ¿Entienden por qué los conceptos son importantes? ¿Saben qué se entiende por conocimiento «estructurado»? ¿Saben por qué es útil estructurar el conocimiento? Comunicar acerca del aprendizaje ayuda a motivar a los estudiantes y a mantenerlos activos en el proceso de aprendizaje.

\section{Prácticas de aula}

Las siguientes prácticas permitirán a los profesores ayudar a los estudiantes a estructurar su conocimiento:

- Dar a los estudiantes muchas oportunidades para la reflexión. La reflexión es una actividad que los profesores y otros profesionales usualmente llevan a cabo por sí mismos, incluso cuando están ocupados en otra actividad. (Por ejemplo, un profesor puede resolver un problema y evaluar el proceso de solución al mismo tiempo.) La mayor parte de los estudiantes no pueden reflexionar sobre la resolución de problemas (o sobre cualquier otra actividad) mientras la llevan a cabo. Tampoco reflexionarán por sí mismos; se les ha de dar tiempo específicamente destinado a la reflexión y se les deben hacer preguntas diseñadas para ayudarlos. Hay muchos contextos sobre los cuales pueden reflexionar los estudiantes: las ideas que surgieron en una actividad o discusión que acaban de terminar; la resolución de problemas; sus estilos de aprendizaje; y el proceso completo de aprendizaje, por nombrar sólo unos pocos. 
- Dar a los estudiantes el tiempo necesario para discutir y pensar sobre la interrelación y priorización de ideas. El conocimiento estructurado está ricamente interconectado y organizado jerárquicamente; las ideas más importantes sirven como conceptos «paraguas» para ideas menos importantes. El conocimiento estructurado conduce a una resolución de problemas eficiente y efectiva, puesto que los conceptos pueden usarse para determinar la aplicabilidad y pertinencia de las ecuaciones, operaciones y procedimientos. El conocimiento estructurado también conduce a una comprensión más profunda.

- Formular problemas en los cuales los rasgos superficiales puedan ser engañosos. Cuando los rasgos superficiales de un problema sugieren un abordaje que no es el más eficaz o efectivo, los estudiantes aprenden que los conceptos son útiles para organizar las ideas. Sin embargo, no es necesario que los problemas sean difíciles; los mejores involucran situaciones que son fáciles de describir y que los estudiantes pueden entender fácilmente. El objetivo es diseñar problemas que requieran un análisis conceptual para ser resueltos.

\section{CONCLUSIÓN}

Para muchos estudiantes, intentar aprender ciencias es una espiral descendente de confusión y fracaso. Las prácticas de aula no se ajustan a sus necesidades; tampoco parecen tener en cuenta sus conocimientos y habilidades. Todos los profesores tienen grandes expectativas para sus estudiantes. Quieren que los estudiantes no sólo entiendan el material, sino que también desarrollen habilidades de pensamiento de orden superior, tales como el análisis, el razonamiento y la resolución de problemas.

Desgraciadamente, las prácticas cotidianas en muchas clases fomentan que los estudiantes aprendan sólo ideas superficiales y habilidades de bajo nivel. Sin unos fundamentos conceptuales fuertes y sin análisis, razonamiento y otras habilidades, los estudiantes continuarán adoptando en la resolución de problemas abordajes superficiales basados en fórmulas.

La resolución de problemas basada en el análisis hace frente a estas cuestiones. Tener en cuenta las ideas previas de los estudiantes permite que ellos distingan entre las definiciones científicas y cotidianas de los conceptos. Hacer que ellos analicen situaciones físicas les ayuda a relacionar los conceptos y mejora aun más su comprensión conceptual. Hacer que razonen sobre situaciones físicas los ayuda a resolver problemas interesantes. Alentarlos para que reflexionen sobre actividades anteriores los ayuda a estructurar su conocimiento.

Ésta es la secuencia de experiencias de aprendizaje a través de la cual los estudiantes adquieren el conocimiento, las habilidades y las oportunidades para tener éxito. Usando el análisis y el razonamiento como un puente entre una comprensión conceptual profunda y una resolución de problemas eficiente, podemos crear un entorno de clase en el cual se favorece el aprendizaje.

Esto no significa que las ecuaciones no sean importantes o útiles. Sólo significa que no son la parte más importante o útil de hacer física. Las ecuaciones son necesarias, pero sólo hacia el final del proceso de resolución de problemas, cuando se aplican principios, leyes y definiciones.

Tampoco significa que los conceptos no sean importantes. Más bien significa que la comprensión conceptual por sí sola no es suficiente para promover las habilidades de resolución de problemas que nosotros, como profesores y didactas de las ciencias, deseamos y valoramos.

Nuestra imagen de una práctica de aula efectiva es muy diferente de las imágenes que mostramos antes. La comunicación es en los dos sentidos; los estudiantes se comunican con el profesor tanto o más que el profesor se comunica con ellos. Los estudiantes son participantes activos en el proceso de aprendizaje -monitorean la comunicación, evalúan su propio aprendizaje, reflexionan acerca de qué experiencias de aprendizaje son más efectivas para ellos, y más cosas. Se autorregulan y son conscientes de los patrones de pensamiento de sus compañeros. Desarrollan una variedad de habilidades, que incluyen las operacionales, procedimentales, estratégicas, de análisis y de razonamiento. Saben cuándo y cómo usarlas. Han aprendido a aprender.

La resolución de problemas basada en el análisis, tal como la hemos presentado aquí, sugiere un abordaje que abandona la tendencia a aprender sin entender - una tendencia que a menudo lleva al fracaso en ciencias. También recupera el entusiasmo por aprender en todos los miembros de la clase.

\section{Nota}

Este artículo ha sido traducido del inglés por Agustín AdúrizBravo. 


\section{REFERENCIAS BIBLIOGRÁFICAS}

ANDERSON, C.W. (1987). Strategic teaching in science, en Jones, B.F., Palincsar, A.S., Ogle, D.S. y Carr, E.G. (eds.). Strategic teaching and learning: Cognitive instruction in the content areas, pp. 73-91. Alexandria, VA: Association for Supervision and Curriculum Development.

BASSOK, M. y HOLYOAK, K.J. (1989). Interdomain transfer between isomorphic topics in algebra and physics. Journal of Experimental Psychology: Learning, Memory and Cognition, 15, pp. 153-166.

BROWN, J.S., COLLINS, A. y DUGUID, P. (1989). Situated cognition and the culture of learning. Educational Researcher, 18, pp. 32-42.

BROWN, D. y CLEMENT, J. (1989). Overcoming misconceptions via analogical reasoning: Factors influencing understanding in a teaching experiment. Instructional Science, 18, pp. 237-261.

CAMP, C. y CLEMENT, J. (1994). Preconceptions in mechanics: Lessons dealing with students' conceptual difficulties. Dubuque, IA: Kendall/Hunt Publishing Company.

CHI, M.T.H., FELTOVICH, P.J. y GLASER, R. (1981). Categorization and representation of physics problems by experts and novices. Cognitive Science, 5, pp. 121-152.

CHI, M.T.H. y GLASER, R. (1981). The measurement of expertise: Analysis of the development of knowledge and skills as a basis for assessing achievement, en Baker, E.L. y Quellmalz, E.S. (eds.).Design, analysis and policy in testing, pp. 37-47. Beverly Hills, CA: Sage Publications.

CLEMENT, J. (1993). Using bridging analogies and anchoring intuitions to deal with students' preconceptions in physics. Journal of Research in Science Teaching, 30(10), pp. 12411257.

DI SESSA, A. (1988). Knowledge in pieces, en Forman, G. y Pufall, P. (eds.). Constructivism in the computer age, pp. 49-70. Hillsdale, Nueva Jersey: Lawrence Erlbaum Associates.

DUFRESNE, R., GERACE, W.J., HARDIMAN,P.T. y MESTRE, J.P. (1992). Constraining novices to perform expert-like problem analyses: Effects on schema acquisition. Journal of the Learning Sciences, 2, pp. 307-331.

DUFRESNE, R.J., GERACE, W.J. y LEONARD, W.J. (1997). Solving physics problems with multiple representations. The Physics Teacher, 35(5), pp. 270-275.

GICK, M.L. y HOLYOAK, K.J. (1980). Analogical problem solving. Cognitive Psychology, 12, pp. 306-355.

GICK, M.L. y HOLYOAK, K.J. (1983). Schema induction and analogical transfer. Cognitive Psychology, 15, pp. 1-38.

GICK, M.L. y HOLYOAK, K.J. (1987). The cognitive basis of knowledge transfer, en Cormier, S.M. y Hagman, J.D. (eds.). Transfer oflearning: Contemporary researchand applications, pp. 9-46. San Diego, CA: Academic Press.

GLASER, R. (1992). Expert knowledge and processes of thinking, en Halpern, D. (ed.). Enhancing thinking skills in the sciences andmathematics, pp.63-75. Hillsdale, Nueva Jersey: Lawrence Erlbaum Associates.

VON GLASERSFELD, E. (1989). Cognition, construction of knowledge, and teaching. Synthese, 80, pp. 121-140.

VON GLASERSFELD, E. (1992). A constructivist's view of learning and teaching, en Duit, R., Goldberg, F. y Niedderer,
H. (eds.). Research in physics learning: Theoretical issues and empirical studies. Proceedings of an International Workshop (Bremen, Alemania, marzo 5-8, 1991), pp. 29-39. Kiel: Institut für die Pädagogik der Naturwissenschaften.

HARDIMAN, P.T., DUFRESNE, R. y MESTRE, J.P. (1989). The relation between problem categorization and problem solving among experts and novices. Memory \& Cognition, 17 , pp. 627-638.

HARMON, M. y MUNGAL, C.F.K. (1992). Science testing and curriculum reform: A report of a study of standardized and text-embedded tests in science. Trabajo presentado en el Annual Meeting of the American Educational Research Association, San Francisco, abril de 1992.

HESTENES, D. y WELLS, M. (1992). A mechanics baseline test. The Physics Teacher, 30(3), pp. 141-158.

HESTENES, D., WELLS, M. y SWACKHAMER, G. (1992). Force concept inventory. The Physics Teacher, 30(3), pp. 159-166.

HEWSON, P.W., KERBY, H.W. y COOK, P.A. (1995). Determining the conceptions of teaching science held by experienced high school science teachers. Journal of Research in Science Teaching, 32, pp. 503-520.

KULM, G. y STUESSY, C. (1991). Assessment in science and mathematics education reform, en Kulm, G. y Malcom, S.M. (eds.). Science assessment in the service of reform, pp. 7187. Washington, DC: American Association for the Advancement of Science.

LARKIN, J.H. (1979). Information processing models in science instruction, en Lochhead, J. y Clement, J. (eds.). Cognitive process instruction, pp. 109-118. Hillsdale, Nueva Jersey: Lawrence Erlbaum Associates.

LARKIN, J.H. (1981). Enriching formal knowledge: A model for learning to solve problems in physics, en Anderson, J.R. (ed.). Cognitive skills and their acquisition, pp. 311-334. Hillsdale, Nueva Jersey: Lawrence Erlbaum Associates.

LARKIN, J.H. (1983). The role of problem representation in physics, en Gentner, D. y Stevens, A.L. (eds.). Mental models, pp. 75-98. Hillsdale, Nueva Jersey: Lawrence Erlbaum Associates.

LARKIN, J.H., McDERMOTT, J., SIMON, D.P. y SIMON, H.A. (1980a). Expert and novice performance in solving physics problems. Science, 208, pp. 1335-1342.

LARKIN, J.H., McDERMOTT, J., SIMON, D.P. y SIMON, H.A. (1980b). Models of competence in solving physics problems. Cognitive Science, 4, pp. 317-345.

LEONARD, W. y GERACE, W. (1996). The power of simple reasoning. The Physics Teacher, 34(5), pp. 280-283.

LEONARD, W.J., DUFRESNE, R.J. y MESTRE, J.P. (1996). Using qualitative problem-solving strategies to highlight the role of conceptual knowledge in solving problems. American Journal of Physics, 64, pp. 1495-1503.

LEONARD, W., DUFRESNE, R., GERACE, W. y MESTRE, J. (1999). Minds-on physics. Activities \& Reader (Vol. 1: Motion; Vol. 2: Interactions; Vol. 3: Conservation Laws \& Concept-Based Problem Solving); Teacher's Guide to accompany Minds-on physics (Vol. 1: Motion; Vol. 2: Interactions; Vol. 3: Conservation Laws \& Concept-Based Problem Solving). Dubuque, IA: Kendall/Hunt Publishing Company. (Para información sobre los materiales curriculares 
Minds-on physics y muestras de actividades, visite la página web del University of Massachusetts Physics Education Research Group, http://umperg.physics.umass.edu/projects/ MindsOnPhysics/.)

LEONARD, W., DUFRESNE, R., GERACE, W. y MESTRE, J. (2000). Minds-on physics. Activities \& reader (Vol. 4 AT: Advanced Topics in Mechanics; Vol. 4 FF: Fundamental Forces \& Fields); Teacher's Guide to accompany Minds-on physics (Vol. 4 FF: Fundamental Forces \& Fields). Dubuque, IA: Kendall/Hunt Publishing Company.

LEONARD, W., DUFRESNE, R., GERACE, W. y MESTRE, J. (2001). Minds-on physics. Activities \& reader (Vol. 4 CS: Complex Systems). Dubuque, IA: Kendall/Hunt Publishing Company.

MALONEY, D.P. (1992). Cognitive physics education research [a bibliography], AAPT Committee on Research in Physics Education. (Se puede pedir a D. Maloney, Physics Department, Indiana University -Purdue University at Fort Wayne, Fort Wayne, IN 46805-1499, USA.)

McDERMOTT, L.C. (1984). Research on conceptual understanding in mechanics. Physics Today, 37(7), pp. 24-32.

McDERMOTT, L.C. y REDISH, E.F. (1999). Resource Letter: PER-1: Physics Education Research. American Journal of Physics, 67, pp. 755-767.

MESTRE, J.P. (1991). Learning and instruction in pre-college physical science. Physics Today, 44(9), pp. 56-62.

MESTRE, J.P. (1994). Cognitive aspects of learning and teaching science, en Fitzsimmons, S.J. y Kerpelman, L.C. (eds.). Teacher enhancement for elementary and secondary science and mathematics: Status, issues and problems, pp. 3-1 y 3-53. Washington, DC: National Science Foundation (NSF 94-80).

MESTRE, J.P., DUFRESNE, R., GERACE, W.J., HARDIMAN, P.T. y TOUGER, J.S. (1993). Promoting skilled problem solving behavior among beginning physics students. Journal of Research in Science Teaching, 30, pp. 303-317.

MESTRE, J.P., GERACE, W.J., DUFRESNE, R.J. y LEONARD, W.J. (1997). Promoting active learning in large classes using a classroom communication system, en Redish, E.F. y Rigden, J.S. (eds.). The changing role of physics departments in modern universities: Proceedings of the International Conference on Undergraduate Physics Education. Part Two: Sample Classes, pp. 1019-1036. Woodbury, NuevaYork: American Institute of Physics.

MINSTRELL, J. (1992). Facets of students' knowledge and relevant instruction, en Duit, R., Goldberg, F. y Niedderer, H. (eds.). Research in physics learning: Theoretical issues and empirical studies. Proceedings of an International Workshop (Bremen, Alemania, marzo 5-8, 1991), pp. 110128. Kiel: IPN.

PFUNDT, H. y DUIT, R. (1991). Bibliography: Students' alternate frameworks in science education. Kiel: IPN.

RESNICK, L.B. (1983). Mathematics and science learning: A new conception. Science, 220, pp. 477-478.

RESNICK, L.B. (1987). Education and learning to think. Washington, DC: National Academy Press.

RITCHIE, S.M., TOBIN, K. y HOOK, K.S. (1997). Teaching referents and the warrants used to test the viability of students' mental models: Is there a link? Journal of Research in Science Teaching, 34, pp. 223-238.

SCHAUBLE, L. (1990). Belief revision in children: The role of prior knowledge and strategies for generating evidence. Journal of Experimental Child Psychology, 49, pp. 31-57.

TOUGER, J.S., DUFRESNE, R.J., GERACE, W.J., HARDIMAN, P.T. y MESTRE, J.P. (1995). How novice physics students deal with explanations. International Journal of Science Education, 17, pp. 255-269.

VAN HEUVELEN, A. (1992). Models of teaching and learning. Sesión plenaria en el Taller sobre Investigación en Didáctica de las Ciencias y las Matemáticas, pp. 20-24. Cathedral Peak, Sudáfrica, enero 1992.

WENK, L., DUFRESNE, R., GERACE, W., LEONARD, W. y MESTRE, J. (1997). Technology-assisted active learning in large lectures, en McNeal, A.P. y D'Avanzo, C. (eds.). Student-active science: Models of innovation in college science teaching, pp. 431-452. Orlando, FL: Saunders College Publishing. 